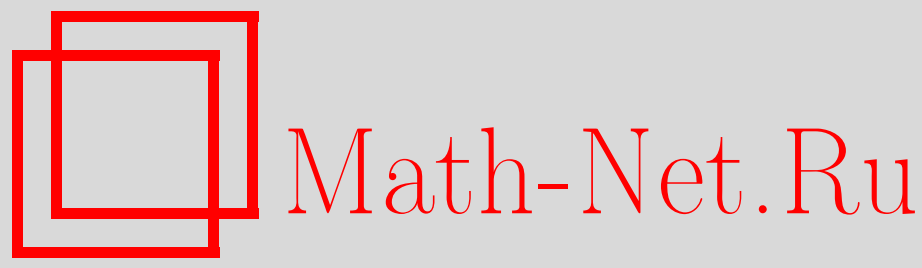

А. Г. Мешков, Векторные гиперболические уравнения, обладающие высшими симметриями, ТМФ, 2009, том 161, номер 2, 176-190

DOI: https://doi.org/10.4213/tmf6430

Использование Общероссийского математического портала Math-Net.Ru подразумевает, что вы прочитали и согласны с пользовательским соглашением http: //www . mathnet.ru/rus/agreement

Параметры загрузки:

IP : 54.224 .135 .184

26 апреля 2023 г., 17:09:14

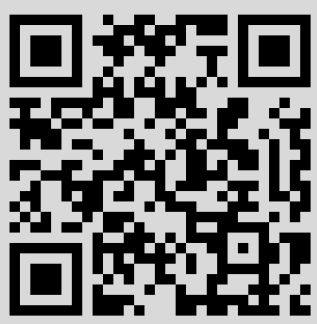




\title{
ВЕКТОРНЫЕ ГИПЕРБОЛИЧЕСКИЕ УРАВНЕНИЯ, ОБЛАДАЮЩИЕ ВЫСШИМИ СИММЕТРИЯМИ
}

\begin{abstract}
Представлен список из одиннадцати векторных гиперболических уравнений, имеющих симметрии третьего порядка по обеим характеристикам. Этот список исчерпывает уравнения, имеющие хотя бы одну симметрию дивергентного вида. Четыре уравнения из списка проинтегрированы явно, одно приведено к линейному виду, еще четыре приведены к нелинейным обыкновенным неавтономным системам. Для шести уравнений найдены преобразования Беклунда.
\end{abstract}

Ключевые слова: гиперболическое уравнение, высшая симметрия, интеграл, преобразование Беклунда, точная интегрируемость.

\section{1. ВВЕДЕНИЕ}

Настоящая работа посвящена изучению нелинейных векторных гиперболических уравнений

$$
\mathbf{u}_{x y}=F_{0} \mathbf{u}+F_{1} \mathbf{u}_{x}+F_{2} \mathbf{u}_{y}
$$

обладающих $S$-интегрируемыми симметриями третьего порядка. Дополнительное ограничение состоит в том, что симметрии имеют потенциальную форму дивергентных уравнений. Вектор-функция $\mathbf{u}$ принимает значения в некотором векторном пространстве $V$ со скалярным произведением $(\cdot, \cdot)$. Коэффициенты $F_{i}$ рассматриваемого уравнения - гладкие скалярные функции, зависящие от всех возможных скалярных произведений трех векторов $\mathbf{u}, \mathbf{u}_{x}, \mathbf{u}_{y}$. Конкретная реализация скалярного произведения может быть любой. Мы используем лишь симметричность, билинейность и дифференцируемость скалярного произведения. Размерность пространства $V$ может быть как конечной, так и бесконечной. Движения пространства $V$ являются, очевидно, симметриями уравнения (1). Такие уравнения будем называть изотропными, а уравнения с нарушенной симметрией относительно движений $V$ назовем анизотропными. Анизотропию можно ввести при помощи второго скалярного произведения $\langle\cdot, \cdot\rangle$. Второе скалярное произведение можно задать, например, в виде $\langle\mathbf{u}, \mathbf{v}\rangle=(\mathbf{u}, L \mathbf{v})$, где $L-$ некоторый линейный самосопряженный оператор

* Орловский государственный технический университет, Орел, Россия.

E-mail: a_meshkov@orel.ru 
такой, что $\partial_{x} L \mathbf{u}=L \mathbf{u}_{x}, \partial_{y} L \mathbf{u}=L \mathbf{u}_{y}$. В конечномерном евклидовом пространстве матрица $L$ постоянна и приводится к диагональному виду.

Таким образом, коэффициенты $F_{i}$ анизотропного уравнения (1) будут зависеть также от дополнительных переменных $\langle\mathbf{u}, \mathbf{u}\rangle,\left\langle\mathbf{u}, \mathbf{u}_{x}\right\rangle, \ldots$

Существует большое число работ, посвященных симметриям и законам сохранения для дифференциальных уравнений (см., например, [1]-[5]). Что касается уравнений вида (1), то нам известна только одна работа [6], в которой исследовались их высшие симметрии. В работе [6] найдены три изотропных уравнения вида (1), два из которых связаны с векторными модифицированными уравнениями Кортевегаде Фриза (мКдФ), и два комплексных изотропных уравнения, симметрии которых бивекторные системы типа систем Шредингера. В настоящей работе мы исследуем уравнения вида (1) без связей. Уравнения со связями будут рассмотрены в другой работе.

На многообразии $\mathfrak{M}$, определенном уравнением (1) и его дифференциальными следствиями, все смешанные производные вектор-функции и выражаются через производные $\partial^{n} \mathbf{u} / \partial x^{n}$ и $\partial^{k} \mathbf{u} / \partial y^{k}$. Поэтому в качестве независимых динамических переменных на многообразии $\mathfrak{M}$ выберем векторы

$$
\mathbf{u}, \quad \mathbf{u}_{x_{n}}=\frac{\partial^{n} \mathbf{u}}{\partial x^{n}}, \quad n>0, \quad \mathbf{u}_{y_{k}}=\frac{\partial^{k} \mathbf{u}}{\partial y^{k}}, \quad k>0
$$

и все их возможные скалярные произведения:

$$
\begin{array}{rlrl}
u_{x x[i, k]} & =\left(\mathbf{u}_{x_{i}}, \mathbf{u}_{x_{k}}\right), & & u_{y y[i, k]}=\left(\mathbf{u}_{y_{i}}, \mathbf{u}_{y_{k}}\right), \quad 0 \leqslant i \leqslant k, \quad k>0, \\
u_{[0,0]} & =(\mathbf{u}, \mathbf{u}), & u_{x y[i, k]}=\left(\mathbf{u}_{x_{i}}, \mathbf{u}_{y_{k}}\right), \quad i>0, \quad k>0, \\
\tilde{u}_{x x[i, k]} & =\left\langle\mathbf{u}_{x_{i}}, \mathbf{u}_{x_{k}}\right\rangle, & & \tilde{u}_{y y[i, k]}=\left\langle\mathbf{u}_{y_{i}}, \mathbf{u}_{y_{k}}\right\rangle, \quad 0 \leqslant i \leqslant k, \quad k>0, \\
\tilde{u}_{[0,0]} & =\langle\mathbf{u}, \mathbf{u}\rangle, & \tilde{u}_{x y[i, k]}=\left\langle\mathbf{u}_{x_{i}}, \mathbf{u}_{y_{k}}\right\rangle, \quad i>0, \quad k>0 .
\end{array}
$$

Если размерность пространства $V$ произвольна, то величины (3) можно считать независимыми переменными. В обозначениях (3) коэффициенты $F_{i}$ анизотропного уравнения могут зависеть от двенадцати переменных

$$
\begin{aligned}
& u_{[0,0]}, u_{x x[0,1]}, u_{x x[1,1]}, u_{y y[0,1]}, u_{y y[1,1]}, u_{x y[1,1]}, \\
& \tilde{u}_{[0,0]}, \tilde{u}_{x x[0,1]}, \tilde{u}_{x x[1,1]}, \tilde{u}_{y y[0,1]}, \tilde{u}_{y y[1,1]}, \tilde{u}_{x y[1,1]},
\end{aligned}
$$

причем в уравнении (1) должны присутствовать оба типа переменных - с тильдами и без них. Если в уравнение входят скалярные переменные только одного типа, то уравнение изотропное.

Определим порядки переменных:

$$
\begin{aligned}
\operatorname{ord} u_{[0,0]} & =\operatorname{ord} \tilde{u}_{[0,0]}=\operatorname{ord} \mathbf{u}=0, & \operatorname{ord} \mathbf{u}_{x_{i}}=i, \\
\operatorname{ord} \mathbf{u}_{y_{k}} & =k, \quad \operatorname{ord} u_{x x[i, k]}=k, & \text { ord } u_{y y[i, j]}=j .
\end{aligned}
$$

Порядок функции $F$ определим как наивысший из порядков переменных, от которых она зависит.

2 Теоретическая и математическая физика, т. 161, № 2, 2009 г. 
Классификация уравнений (1), обладающих высшими симметриями, проводилась с точностью до точечных преобразований. Возможны следующие точечные преобразования.

1. Масштабные преобразования:

$$
x^{\prime}=\alpha x, \quad y^{\prime}=\beta t, \quad \mathbf{u}^{\prime}=\gamma \mathbf{u} .
$$

2. Переобозначение независимых переменных $y \leftrightarrow x$.

3. Линейное преобразование метрик в анизотропных уравнениях:

$$
\langle\mathbf{u}, \mathbf{v}\rangle \rightarrow \alpha\langle\mathbf{u}, \mathbf{v}\rangle+\beta(\mathbf{u}, \mathbf{v}), \quad(\mathbf{u}, \mathbf{v}) \rightarrow \gamma\langle\mathbf{u}, \mathbf{v}\rangle+\delta(\mathbf{u}, \mathbf{v})
$$

где $\alpha, \beta, \gamma$ и $\delta$ - постоянные.

4. Если уравнение (1) однородно, т.е.

$$
F_{k}\left(\lambda \mathbf{u}, \lambda \mathbf{u}_{x}, \lambda \mathbf{u}_{y}\right)=F_{k}\left(\mathbf{u}, \mathbf{u}_{x}, \mathbf{u}_{t}\right) \quad \forall \lambda \in \mathbb{R}, \quad k=0,1,2,
$$

то допускается калибровочное преобразование:

$$
\mathbf{u}^{\prime}=\mathbf{u} e^{a x+b y}, \quad a \in \mathbb{R}, \quad b \in \mathbb{R} .
$$

5. Преобразование модуля вектор-функции:

$$
\mathbf{v}=\mathbf{u} f\left(u_{[0,0]}, \tilde{u}_{[0,0]}\right) \text {. }
$$

Это преобразование обратимо тогда и только тогда, когда уравнения

$$
v_{[0,0]}=u_{[0,0]} f^{2}\left(u_{[0,0]}, \tilde{u}_{[0,0]}\right), \quad \tilde{v}_{[0,0]}=\tilde{u}_{[0,0]} f^{2}\left(u_{[0,0]}, \tilde{u}_{[0,0]}\right)
$$

разрешимы относительно $u_{[0,0]}$ и $\tilde{u}_{[0,0]}$.

\section{2. РЕЗУЛЬТАТЫ КЛАССИФИКАЦИИ}

Проблема классификации гиперболических уравнений, обладающих высшими симметриями, не решена в общей постановке даже в скалярном случае из-за трудностей принципиального характера (см., например, [7]). Наши результаты также не претендуют на полноту. В настоящей работе представлены гиперболические уравнения, обладающие заданными $x$-симметриями третьего порядка, причем симметрии имеют потенциальную форму дивергентных уравнений:

$$
\begin{aligned}
& \mathbf{u}_{t}=\mathbf{u}_{x x x}+\frac{3}{2}\left(\frac{a^{2}\left(\mathbf{u}_{x}, \mathbf{u}_{x x}\right)^{2}}{1+a \mathbf{u}_{x}^{2}}-a \mathbf{u}_{x x}^{2}\right) \mathbf{u}_{x} \\
& \mathbf{u}_{t}=\mathbf{u}_{x x x}-3 \frac{\left(\mathbf{u}_{x}, \mathbf{u}_{x x}\right)}{\mathbf{u}_{x}^{2}} \mathbf{u}_{x x}+\frac{3}{2}\left(\frac{\mathbf{u}_{x x}^{2}}{\mathbf{u}_{x}^{2}}+\frac{\left(\mathbf{u}_{x}, \mathbf{u}_{x x}\right)^{2}}{\mathbf{u}_{x}^{4}\left(1+a \mathbf{u}_{x}^{2}\right)}\right) \mathbf{u}_{x} \\
& \mathbf{u}_{t}=\mathbf{u}_{x x x}-\frac{3}{2}(p+1) \frac{\left(\mathbf{u}_{x}, \mathbf{u}_{x x}\right)}{p \mathbf{u}_{x}^{2}} \mathbf{u}_{x x}+\frac{3}{2}(p+1)\left(\frac{\mathbf{u}_{x x}^{2}}{\mathbf{u}_{x}^{2}}-\frac{a\left(\mathbf{u}_{x}, \mathbf{u}_{x x}\right)^{2}}{p^{2} \mathbf{u}_{x}^{2}}\right) \mathbf{u}_{x} \\
& \mathbf{u}_{t}=\mathbf{u}_{x x x}-3 \frac{\left(\mathbf{u}_{x}, \mathbf{u}_{x x}\right)}{\mathbf{u}_{x}^{2}} \mathbf{u}_{x x}+3 \frac{\mathbf{u}_{x x}^{2}}{\mathbf{u}_{x}^{2}} \mathbf{u}_{x}
\end{aligned}
$$




$$
\begin{aligned}
& \mathbf{u}_{t}=\mathbf{u}_{x x x}-3 \frac{\left(\mathbf{u}_{x}, \mathbf{u}_{x x}\right)}{\mathbf{u}_{x}^{2}} \mathbf{u}_{x x}+\frac{3}{2}\left(\frac{\mathbf{u}_{x x}^{2}}{\mathbf{u}_{x}^{2}}+\frac{\left(\mathbf{u}_{x}, \mathbf{u}_{x x}\right)^{2}}{\mathbf{u}_{x}^{4}}-\frac{\left\langle\mathbf{u}_{x}, \mathbf{u}_{x}\right\rangle}{\mathbf{u}_{x}^{2}}\right) \mathbf{u}_{x}, \\
& \mathbf{u}_{t}=\mathbf{u}_{x x x}-3 \frac{\left(\mathbf{u}_{x x}, \mathbf{u}_{x}\right)}{\mathbf{u}_{x}^{2}} \mathbf{u}_{x x}+\frac{3}{2} \frac{\mathbf{u}_{x x}^{2}}{\mathbf{u}_{x}^{2}} \mathbf{u}_{x}
\end{aligned}
$$

где $p=\sqrt{1+a \mathbf{u}_{x}^{2}}, a$ - постоянная. Здесь и далее применяются обычные векторные обозначения, в частности сокращения $\mathbf{u}^{2}=(\mathbf{u}, \mathbf{u}),|\mathbf{u}|=\sqrt{\mathbf{u}^{2}}$.

Список уравнений (10)-(15) является полным и получен в работе [8]. Доказано, что уравнения (10)-(15) удовлетворяют достаточно большому числу условий интегрируемости, имеют высшие законы сохранения, симметрии пятого порядка и преобразования Беклунда.

Вероятно, существуют гиперболические уравнения, обладающие симметриями более высокого порядка и не имеющие симметрий третьего порядка. В настоящей работе этот вопрос не рассматривается ввиду его сложности.

ОСНовнаЯ ТЕОРЕма. Если гиперболическое уравнение (1) допускает интегрируемые симметрии третьего порядка по каждой характеристике и если при этом хотя бы одна из симметрий принадлежит списку (10)-(15), то такое уравнение приводится точечными преобразованиями (5)-(8) к одному из следующих уравнений:

$$
\begin{aligned}
& \mathbf{u}_{x y}=\sqrt{1+a \mathbf{u}_{x}^{2}} \mathbf{u} \\
& \mathbf{u}_{x y}=(p-1) \mathbf{u}+(p+1) \frac{\left(\mathbf{u}, \mathbf{u}_{x}\right)}{\mathbf{u}_{x}^{2}} \mathbf{u}_{x}, \quad p=\sqrt{1+a \mathbf{u}_{x}^{2}}, \quad a \neq 0, \\
& \mathbf{u}_{x y}=\left|\mathbf{u}_{x}\right|^{-1} \sqrt{1+a \mathbf{u}_{x}^{2}} \mathbf{u}_{x}, \\
& \mathbf{u}_{x y}=\left(c+\left|\mathbf{u}_{x}\right|^{-1}\right) \mathbf{u}_{x}, \\
& \mathbf{u}_{x y}=\frac{\left(\mathbf{u}, \mathbf{u}_{y}\right)+\varphi}{\mathbf{u}^{2}+b} \mathbf{u}_{x}, \quad \varphi=\sqrt{\left(\mathbf{u}, \mathbf{u}_{y}\right)^{2}-\left(\mathbf{u}_{y}^{2}+a\right)\left(\mathbf{u}^{2}+b\right)}, \\
& \mathbf{u}_{x y}=\frac{\chi+\psi}{\mathbf{u}^{2}} \mathbf{u}_{x}, \quad \chi=\left(\mathbf{u}, \mathbf{u}_{y}\right)+a, \quad \psi=\sqrt{\chi^{2}-\mathbf{u}^{2} \mathbf{u}_{y}^{2}}, \\
& \mathbf{u}_{x y}=\left(a+\frac{b\left(\mathbf{u}_{y}, \mathbf{u}_{x}\right)+c\left(\mathbf{u}, \mathbf{u}_{x}\right)}{\mathbf{u}_{x}^{2}}\right) \mathbf{u}_{x}, \\
& \mathbf{u}_{x y}=\left|\mathbf{u}_{y}\right|^{-1} \sqrt{1+a \mathbf{u}_{x}^{2}} \mathbf{u}_{y}, \quad a \neq 0, \\
& \mathbf{u}_{x y}=\left|\mathbf{u}_{x}\right|^{-1} \sqrt{a+\left\langle\mathbf{u}_{y}, \mathbf{u}_{y}\right\rangle} \mathbf{u}_{x}, \\
& \mathbf{u}_{x y}=\frac{\left(\mathbf{u}, \mathbf{u}_{y}\right)}{a+\mathbf{u}^{2}} \mathbf{u}_{x} \\
& \mathbf{u}_{x y}=2 \frac{\left(\mathbf{u}, \mathbf{u}_{x}\right) \mathbf{u}_{y}-\left(\mathbf{u}_{y}, \mathbf{u}_{x}\right) \mathbf{u}}{\mathbf{u}^{2}+a}+\frac{(1+2 k q)\left(\mathbf{u}, \mathbf{u}_{y}\right)+b \eta}{r} \mathbf{u}_{x}, \\
& b=\sqrt{1+4 a k}, \quad \eta=\sqrt{\left(\mathbf{u}, \mathbf{u}_{y}\right)^{2}-r\left(\mathbf{u}_{y}^{2}+c q^{2}\right)}, \\
& r=\mathbf{u}^{2}+k\left(\mathbf{u}^{2}+a\right)^{2}, \quad q=\mathbf{u}^{2}+a, \quad a \neq 0,
\end{aligned}
$$

где $a, b, c, k-$ постоянные. 
ЗАмечАние 1. Уравнения (16) и (17) были найдены ранее в работе [6] по их полиномиальным симметриям. Симметрии по второй характеристике в этой работе не вычислялись.

ЗАмЕчАниЕ 2. Уравнения (18)-(21) интегрируются в квадратурах; уравнение (22) приводится к линейному уравнению с переменными коэффициентами; уравнения (23)-(26) приводятся к нелинейным обыкновенным неавтономным системам уравнений.

ЗАмЕчАНиЕ 3. Чтобы проиллюстрировать характер точечных преобразований, встречавшихся в процессе классификации, приведем один пример. Уравнение

$$
\mathbf{u}_{x y}=\frac{2\left(\mathbf{u}, \mathbf{u}_{y}\right)}{\mathbf{u}^{2}-c} \mathbf{u}_{x}+\frac{2}{\mathbf{u}^{2}+c}\left(\left(\mathbf{u}, \mathbf{u}_{x}\right) \mathbf{u}_{y}-\left(\mathbf{u}_{y}, \mathbf{u}_{x}\right) \mathbf{u}\right)
$$

приводится подстановкой

$$
\mathbf{u}=\frac{1+\sqrt{1+4 c \mathbf{v}^{2}}}{2 \mathbf{v}^{2}} \mathbf{v}
$$

к следующему виду:

$$
\mathbf{v}_{x y}=\frac{4 c\left(\mathbf{v}, \mathbf{v}_{x}\right)}{1+4 c \mathbf{v}^{2}} \mathbf{v}_{y} .
$$

При $c \neq 0$ это уравнение сводится к (25), а при $c=0$ принимает вид уравнения Даламбера. Таким образом, при $c=0$ уравнение $(27)$ интегрируется в квадратурах при помощи преобразования инверсии $\mathbf{u}=\mathbf{v} / \mathbf{v}^{2}$.

\section{3. СОКРАЩЕННОЕ ДОКАЗАТЕЛЬСТВО ОСНОВНОЙ ТЕОРЕМЫ}

Рассмотрим симметрию третьего порядка уравнения (1) в наиболее общем виде:

$$
\mathbf{u}_{t}=\mathbf{S}, \quad \mathbf{S}=f_{1} \mathbf{u}_{x}+f_{2} \mathbf{u}_{x x}+f_{3} \mathbf{u}_{x x x}+g_{1} \mathbf{u}_{y}+g_{2} \mathbf{u}_{y y}+g_{3} \mathbf{u}_{y y y}+g_{0} \mathbf{u},
$$

где $f_{i}, g_{k}$ - гладкие скалярные функции переменных (3). Для упрощения задачи введем только одно ограничение: порядок всех функций в (28) не должен быть выше второго. Обозначим правую часть уравнения (1) как $\mathbf{H}(\mathbf{u})=F_{0} \mathbf{u}+F_{1} \mathbf{u}_{x}+F_{2} \mathbf{u}_{y}$, тогда уравнение для симметрий принимает следующий вид:

$$
\left.\left(\mathbf{H}_{*}(\mathbf{u})-D_{x} D_{y}\right) \mathbf{S}\right|_{\mathbf{u}_{x y}=\mathbf{H}}=0
$$

где $D_{x}$ и $D_{y}$ - операторы полных производных по $x$ и $y$ соответственно,

$$
\mathbf{H}_{*}(\mathbf{u}) \mathbf{S}=\left.\frac{\partial}{\partial \varepsilon} \mathbf{H}(\mathbf{u}+\varepsilon \mathbf{S})\right|_{\varepsilon=0}=\left[\left(\mathbf{S}, \nabla_{u}\right)+\left(D_{y} \mathbf{S}, \boldsymbol{\nabla}_{u_{y}}\right)+\left(D_{x} \mathbf{S}, \boldsymbol{\nabla}_{u_{x}}\right)\right] \mathbf{H}
$$

Явный вид уравнения (29) сложный, поэтому мы его опускаем. Отметим только, что уравнение (29) можно получить как условие совместности двух следующих уравнений:

$$
\mathbf{u}_{x y}=\mathbf{H}, \quad \mathbf{u}_{t}=\mathbf{S}
$$

где $t$ - параметр эволюции. Именно поэтому мы записываем симметрии как эволюционные уравнения. 
Переход на многообразие $\mathbf{u}_{x y}=\mathbf{H}$ в (29) не столь тривиален, поэтому рассмотрим этот вопрос подробнее. Ключевыми являются формулы дифференцирования векторных переменных на многообразии:

$$
D_{x} \mathbf{u}_{x_{n}}=\mathbf{u}_{x_{n+1}}, \quad D_{y} \mathbf{u}_{y_{n}}=\mathbf{u}_{y_{n+1}}, \quad D_{y} \mathbf{u}_{x_{n}}=\mathbf{R} \mathbf{x}[n], \quad D_{x} \mathbf{u}_{y_{n}}=\mathbf{R y}[n] .
$$

При этом, очевидно,

$$
\mathbf{R x}[1]=\mathbf{R y}[1]=\mathbf{u}_{x y}=\mathbf{H} .
$$

Далее, в силу коммутативности операторов $D_{x}$ и $D_{y}$ имеем $D_{x} \mathbf{R} \mathbf{x}[n]=D_{y} \mathbf{u}_{x_{n+1}}=$ $\mathbf{R} \mathbf{x}[n+1]$. Аналогично получаем рекуррентное соотношение для $\mathbf{R} \mathbf{y}[n]$. Пара соотношений

$$
\mathbf{R x}[n+1]=D_{x} \mathbf{R x}[n], \quad \mathbf{R y}[n+1]=D_{y} \mathbf{R y}[n]
$$

с начальными условиями (30) позволяет вычислять коэффициентные функции $\mathbf{R} \mathbf{x}[n]$ и $\mathbf{R y}[n]$ при помощи самих операторов $D_{x}$ и $D_{y}$, так как на каждом шаге приходится дифференцировать переменные, действие на которые уже определено на предыдущих шагах. Дифференцирование скалярных переменных по $x$ производится согласно следующим формулам:

$$
\begin{aligned}
D_{x} u_{x x[i, k]} & =u_{x x[i+1, k]}+u_{x x[i, k+1]}, \quad i<k, \\
D_{x} u_{x x[i, i]} & =2 u_{x x[i, i+1]}, \quad D_{x} u_{[0,0]}=2 u_{x x[0,1]}, \\
D_{x} u_{x y[i, k]} & =u_{x y[i+1, k]}+\left(\mathbf{u}_{x_{i}}, \mathbf{R y}[k]\right), \\
D_{x} u_{y y[i, k]} & =\left(\mathbf{u}_{y_{i}}, \mathbf{R y}[k]\right)+\left(\mathbf{u}_{y_{k}}, \mathbf{R y}[i]\right) .
\end{aligned}
$$

Для оператора $D_{y}$ легко получить аналогичные формулы, а формулы для переменных с тильдами полностью аналогичны приведенным, поэтому мы их опускаем.

Следующая лемма устанавливает структуру симметрий третьего порядка.

Лемма 1. Симметрия третьего порядка системы (1) имеет следующий вид:

$$
\mathbf{u}_{t}=f_{3} \mathbf{u}_{x x x}+f_{2} \mathbf{u}_{x x}+f_{1} \mathbf{u}_{x}+f_{0} \mathbf{u}+g_{3} \mathbf{u}_{y y y}+g_{2} \mathbf{u}_{y y}+g_{1} \mathbf{u}_{y}+g_{0} \mathbf{u}+h_{0} \mathbf{u},
$$

где функиии $f_{i}$ зависят от $\mathbf{u}$ и ее производных только по $x$, а функиии $g_{i}$ зависят от $\mathbf{u}$ u ее производных толъко по у. Функция $h_{0}$ может зависеть только от следующих переменных нулевого и первого порядков:

$$
u_{[0,0]}, \tilde{u}_{[0,0]}, u_{x x[i, 1]}, \tilde{u}_{x x[j, 1]}, u_{y y[k, 1]}, \tilde{u}_{y y[l, 1]}, \quad i, j, k, l=0,1 .
$$

Доказательство получается путем расщепления определяющего уравнения (29) по векторным $\mathbf{u}, \mathbf{u}_{x_{i}}, \mathbf{u}_{y_{i}}$ и скалярным переменным. При этом коэффициенты $f_{1}$, $f_{2}, f_{3}, g_{1}, g_{2}, g_{3}$ в (28) определяются из независимых уравнений, причем $f_{3}$ и $g_{3}$ могут быть постоянными. Из оставшихся связанных уравнений определяются $f_{0}, g_{0}$ и $h_{0}$.

Если предположить, что $h_{0}=0$, то уравнения для коэффициентов $f_{i}$ становятся независимыми от уравнений для коэффициентов $g_{i}$. Это означает, что симметрия (31) распадается на две независимые симметрии

$$
\begin{aligned}
& \mathbf{u}_{t}=\mathbf{u}_{x x x}+f_{2} \mathbf{u}_{x x}+f_{1} \mathbf{u}_{x}+f_{0} \mathbf{u}, \\
& \mathbf{u}_{t}=\mathbf{u}_{y y y}+g_{2} \mathbf{u}_{y y}+g_{1} \mathbf{u}_{y}+g_{0} \mathbf{u}
\end{aligned}
$$


где функции $f_{i}$ зависят только от $\mathbf{u}, \mathbf{u}_{x}$ и $\mathbf{u}_{x x}$, а функции $g_{i}-$ от $\mathbf{u}, \mathbf{u}_{y}$ и $\mathbf{u}_{y y}$. Подчеркнем, что здесь речь идет только о высших симметриях, не зависящих явно от $x$ и $y$. Существуют нераспадающиеся симметрии первого порядка, например $\mathbf{S}=y \mathbf{u}_{y}-x \mathbf{u}_{x}-$ генератор преобразований Лоренца. Здесь мы полагаем $f_{3}=g_{3}=1$, потому что только такие $S$-интегрируемые уравнения известны в настоящее время. Симметрия (32) называется $x$-симметрией, а (33) - $y$-симметрией. Далее рассматриваем только $x$-симметрии и $y$-симметрии, а не симметрии общего вида $(31)$.

Сначала рассмотрим гиперболические уравнения (1), обладающие одной $x$-симметрией из списка (10)-(15). При решении определяющего уравнения возникают две квазилинейные системы, решения которых записываются только в неявной форме. Первая система

$$
\begin{array}{rlrl}
\frac{\partial h}{u_{y y[0,1]}} & =-2 h \frac{\partial h}{\partial u_{y y[1,1]}}, & \frac{\partial h}{u_{[0,0]}} & =h^{2} \frac{\partial h}{\partial u_{y y[1,1]}}, \\
\frac{\partial h}{\tilde{u}_{y y[0,1]}}=-2 h \frac{\partial h}{\partial \tilde{u}_{y y[1,1]}}, & \frac{\partial h}{\tilde{u}_{[0,0]}} & =h^{2} \frac{\partial h}{\partial \tilde{u}_{y y[1,1]}}
\end{array}
$$

определяет функцию $h=h\left(u_{[0,0]}, u_{y y[0,1]}, u_{y y[1,1]}, \tilde{u}_{[0,0]}, \tilde{u}_{y y[0,1]}, \tilde{u}_{y y[1,1]}\right)$ и, очевидно, совместна. Вторая система определяет две функции $h_{i}=h_{i}\left(u_{[0,0]}, u_{y y[0,1]}, u_{y y[1,1]}\right)$, $i=1,2$ :

$$
\begin{gathered}
2 \frac{\partial h_{1}}{\partial u_{[0,0]}}+\left(h_{1}+h_{2} u_{y y[0,1]}\right) \frac{\partial h_{1}}{\partial u_{y y[0,1]}}+2 h_{2} u_{y y[1,1]} \frac{\partial h_{1}}{\partial u_{y y[1,1]}}-h_{1} h_{2}=0, \\
\left(1-u_{[0,0]} h_{2}\right) \frac{\partial h_{1}}{\partial u_{y y[0,1]}}+2\left(h_{1}-h_{2} u_{y y[0,1]}\right) \frac{\partial h_{1}}{\partial u_{y y[1,1]}}+h_{2}=0 \\
2 \frac{\partial h_{2}}{\partial u_{[0,0]}}+\left(h_{1}+h_{2} u_{y y[0,1]}\right) \frac{\partial h_{2}}{\partial u_{y y[0,1]}}+2 h_{2} u_{y y[1,1]} \frac{\partial h_{2}}{\partial u_{y y[1,1]}}+h_{2}^{2}=0 \\
\left(1-u_{[0,0]} h_{2}\right) \frac{\partial h_{2}}{\partial u_{y y[0,1]}}+2\left(h_{1}-h_{2} u_{y y[0,1]}\right) \frac{\partial h_{2}}{\partial u_{y y[1,1]}}=0
\end{gathered}
$$

эта система совместна при $1-u_{[0,0]} h_{2} \neq 0$.

Лемма 2. Гиперболическое уравнение вида (1), допускающее какую-либо $x$-симметрию вида (10)-(15), принадлежит следующему списку:

$$
\begin{aligned}
& \mathbf{u}_{x y}=\sqrt{1+a \mathbf{u}_{x}^{2}} \mathbf{u}, \\
& \mathbf{u}_{x y}=\left|\mathbf{u}_{y}\right|^{-1} \sqrt{1+a \mathbf{u}_{x}^{2}} \mathbf{u}_{y}, \\
& \mathbf{u}_{x y}=\left|\mathbf{u}_{x}\right|^{-1} \sqrt{1+a \mathbf{u}_{x}^{2}} \mathbf{u}_{x}, \\
& \mathbf{u}_{x y}=\left(c+\left|\mathbf{u}_{x}\right|^{-1} \sqrt{c_{1}\left(\mathbf{u}_{y}-c \mathbf{u}\right)^{2}+c_{2}}\right) \mathbf{u}_{x}, \\
& \mathbf{u}_{x y}=(p-1) \mathbf{u}+(p+1) \frac{\left(\mathbf{u}, \mathbf{u}_{x}\right)}{\mathbf{u}_{x}^{2}} \mathbf{u}_{x}, \quad p=\sqrt{1+a \mathbf{u}_{x}^{2}}, \quad a \neq 0, \\
& \left.\mathbf{u}_{x y}=\left(a+\frac{b\left(\mathbf{u}_{y}, \mathbf{u}_{x}\right)+c\left(\mathbf{u}, \mathbf{u}_{x}\right)}{\mathbf{u}_{x}^{2}}\right) \mathbf{u}_{x}, \quad \mathbf{u}, \quad \mathbf{u}^{2}\right) \mathbf{u}_{x}, \\
& \mathbf{u}_{x y}=\left(c+\left|\mathbf{u}_{x}\right|^{-1} \sqrt{\left\langle\mathbf{u}_{y}-c \mathbf{u}, \mathbf{u}_{y}-c \mathbf{u}\right\rangle+b\left(\mathbf{u}_{y}-c \mathbf{u}\right)^{2}+c},\right.
\end{aligned}
$$




$$
\begin{aligned}
& \mathbf{u}_{x y}=h\left(u_{[0,0]}, u_{y y[0,1]}, u_{y y[1,1]}, \tilde{u}_{[0,0]}, \tilde{u}_{y y[0,1]}, \tilde{u}_{y y[1,1]}\right) \mathbf{u}_{x} \\
& \mathbf{u}_{x y}=h_{1} \mathbf{u}_{x}+h_{2}\left(\left(\mathbf{u}, \mathbf{u}_{x}\right) \mathbf{u}_{y}-\left(\mathbf{u}_{y}, \mathbf{u}_{x}\right) \mathbf{u}\right)
\end{aligned}
$$

где $a, b, c, c_{1}, c_{2}$ - постоянные, функиия $h$ удовлетворяет системе (34), а функиии $h_{1}$ и $h_{2}$ - системе (35).

Доказательство получается прямым вычислением. Уравнения (36) и (37) имеют общую симметрию (10); симметрия уравнения (38) имеет вид (11), где $a \neq 0$; уравнение (39) допускает симметрию (11), где $a=0$; уравнение (40) допускает симметрию (12), а уравнение (41) - симметрию (13); анизотропное уравнение (42) обладает симметрией (14); уравнение (43) допускает пять симметрий: (15), (10) и (11), где $a=0$, а также (13) и (14). Последнее эквивалентно тому, что уравнение (43) имеет следующий набор простейших симметрий:

$$
\begin{aligned}
& \mathbf{S}_{1}=\mathbf{u}_{x x x}, \quad \mathbf{S}_{2}=\frac{\left(\mathbf{u}_{x}, \mathbf{u}_{x x}\right)}{\mathbf{u}_{x}^{2}} \mathbf{u}_{x x}, \quad \mathbf{S}_{3}=\frac{\mathbf{u}_{x x}^{2}}{\mathbf{u}_{x}^{2}} \mathbf{u}_{x} \\
& \mathbf{S}_{4}=\frac{\left(\mathbf{u}_{x}, \mathbf{u}_{x x}\right)^{2}}{\mathbf{u}_{x}^{4}} \mathbf{u}_{x}, \quad \mathbf{S}_{5}=\frac{\left\langle\mathbf{u}_{x}, \mathbf{u}_{x}\right\rangle}{\mathbf{u}_{x}^{2}} \mathbf{u}_{x}
\end{aligned}
$$

Наконец, семейство уравнений (44) допускает симметрию (15).

ЗАмЕчАниЕ 4. В уравнении (42) без ограничения общности можно положить $b=0$, так как эта постоянная уничтожается путем преобразования второй метрики $\langle\cdot, \cdot\rangle \rightarrow\langle\cdot, \cdot\rangle-b(\cdot, \cdot)$.

ЗАмЕчАниЕ 5. Если уравнение (43) изотропно, то оно является частным случаем уравнения (44). Поэтому следует считать, что правая часть уравнения (43) зависит от переменных обоих типов.

Далее вычислим $y$-симметрии для каждого из уравнений (36)-(44).

Для уравнений (36)-(42), не содержащих произвольных функций, удается найти $y$-симметрии без дополнительных предположений относительно функций $g_{i}$. Уравнения (43) и (44) обладают нетривиальными интегралами, поэтому они имеют симметрии с функциональным произволом. По этой причине функции $g_{1}$ и $g_{2}$ были подчинены первым двум условиям интегрируемости (см. [8], [9]). Эти условия сводятся к тому, что функция $g_{1}$ должна быть квадратичной по $\mathbf{u}_{y y}$, a $g_{2}=D_{y} \chi\left(\mathbf{u}, \mathbf{u}_{y}\right)$.

В результате всех перечисленных вычислений и исключения нескольких уравнений, сводящихся к другим уравнениям или к уравнению Даламбера, получаем список (16)-(26). Интегрируемость в квадратурах и редукции к обыкновенным уравнениям рассмотрены ниже.

Интегралы и решения уравнений. Восемь уравнений из списка (16)-(26) имеют вид $\mathbf{u}_{x y}=f \mathbf{u}_{x}$ (уравнение (23) отличается заменой $y \leftrightarrow x$ ). Нетрудно проверить, что каждое из этих уравнений имеет векторный $y$-интеграл:

$$
D_{y} \frac{\mathbf{u}_{x}}{\left|\mathbf{u}_{x}\right|}=0 .
$$

Отсюда находим, что

$$
\mathbf{u}_{x}=\left|\mathbf{u}_{x}\right| \boldsymbol{\Phi}(x),
$$


где $\boldsymbol{\Phi}$ - произвольная вектор-функция, принимающая значения на сфере: $\boldsymbol{\Phi}^{2}=1$.

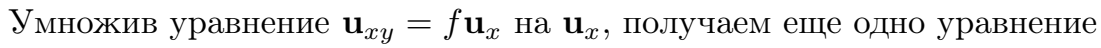

$$
\left(\ln \left|\mathbf{u}_{x}\right|\right)_{y}=f .
$$

1. Уравнение (18) имеет векторный $y$-интеграл вида (45), что приводит к формуле (46). При этом уравнение (47) принимает вид $\left|\mathbf{u}_{x}\right|_{y}=\sqrt{1+a\left|\mathbf{u}_{x}\right|^{2}}$. Отсюда находим решение уравнения (18) при помощи одной квадратуры:

$$
\mathbf{u}(x, y)=a^{-1 / 2} \int \boldsymbol{\Phi}(x) \operatorname{sh}\left[a^{1 / 2}(y+\varphi(x))\right] d x+\boldsymbol{\Psi}(y), \quad \boldsymbol{\Phi}^{2}=1,
$$

где $\boldsymbol{\Psi}$ - произвольная вектор-функция, $\varphi$ - произвольная скалярная функция. В решении можно перейти к пределу $a \rightarrow 0$.

2. Уравнение (19) интегрируется аналогично предыдущему:

$$
\mathbf{u}(x, y)=\int \boldsymbol{\Phi}(x) e^{c y+c \varphi(x)} d x+\boldsymbol{\Psi}(y), \quad \boldsymbol{\Phi}^{2}=1, \quad c=\text { const } .
$$

3. Уравнение (20) имеет векторный $y$-интеграл вида (45), но более сильные следствия вытекают из существования скалярного $x$-интеграла:

$$
\frac{\left(\mathbf{u}, \mathbf{u}_{y}\right)+\varphi}{\mathbf{u}^{2}+b}=\frac{f^{\prime}(y)}{f(y)}
$$

Это позволяет проинтегрировать уравнение (20) в квадратурах:

$$
\mathbf{u}(x, y)=f(y) \boldsymbol{\Phi}(x)+\mathbf{\Psi}(y)
$$

где $\boldsymbol{\Phi}$ и $\boldsymbol{\Psi}$ - произвольные вектор-функции, а $f$ определяется из уравнения (48), которое приводится к следующему виду:

$$
f^{\prime 2}\left(\Psi^{2}+b\right)-2 f f^{\prime}\left(\Psi, \Psi^{\prime}\right)+f^{2}\left(\Psi^{\prime 2}+a\right)=0
$$

4. Уравнение (21), как и предыдущее, имеет скалярный $x$-интеграл

$$
\frac{\left(\mathbf{u}, \mathbf{u}_{y}\right)+a+\psi}{\mathbf{u}^{2}}=\frac{f^{\prime}(y)}{f(y)}
$$

поэтому его общее решение имеет вид (49), где функция $f$ определяется из уравнения

$$
f^{\prime 2} \Psi^{2}-2 f f^{\prime}\left(\left(\boldsymbol{\Psi}, \Psi^{\prime}\right)+a\right)+f^{2} \Psi^{\prime 2}=0
$$

вытекающего из (50).

5. Уравнение (22) имеет $y$-интеграл (45), поэтому $\mathbf{u}_{x}=\boldsymbol{\Phi}(x)\left|\mathbf{u}_{x}\right|$ и $\left|\mathbf{u}_{x}\right|=\left(\boldsymbol{\Phi}, \mathbf{u}_{x}\right)$. Подставив в правую часть уравнения (22) эти выражения, приводим его к линейному виду

$$
\mathbf{u}_{x y}=\boldsymbol{\Phi}(x)\left(a\left(\boldsymbol{\Phi}, \mathbf{u}_{x}\right)+b\left(\boldsymbol{\Phi}, \mathbf{u}_{y}\right)+c(\boldsymbol{\Phi}, \mathbf{u})\right), \quad \boldsymbol{\Phi}^{2}=1 .
$$

Поскольку $\mathbf{\Phi}$ не зависит от $y$, вектор-функции $\mathbf{u}_{y}, \mathbf{u}_{y y}, \mathbf{u}_{y y y}, \ldots$ являются симметриями уравнения (51). Можно проверить, что равносильное уравнение (22) также допускает эти симметрии. 
6. Уравнение (23) имеет векторный $x$-интеграл $D_{x}\left(\mathbf{u}_{y} /\left|\mathbf{u}_{y}\right|\right)=0$ и сводится к системе

$$
\mathbf{u}_{y}=w \boldsymbol{\Phi}(y), \quad w_{x}=\sqrt{1+a \mathbf{u}_{x}^{2}}, \quad \boldsymbol{\Phi}^{2}(y)=1 .
$$

Уравнение не имеет векторных $y$-интегралов, поэтому линеаризовать его не удается. Если положить $\mathbf{u}_{x}=r \mathbf{v}, \mathbf{v}^{2}=1$, то получаем систему обыкновенных неавтономных уравнений для $r$ и $\mathbf{v}$ :

$$
\begin{aligned}
& \mathbf{u}_{y}=w \boldsymbol{\Phi}(y), \quad \mathbf{u}_{x}=r \mathbf{v}, \quad w_{x}=\sqrt{1+a r^{2}} \\
& r_{y}=(\mathbf{v}, \boldsymbol{\Phi}) \sqrt{1+a r^{2}}, \quad \mathbf{v}_{y}=r^{-1} \sqrt{1+a r^{2}}[\mathbf{\Phi}-\mathbf{v}(\mathbf{v}, \boldsymbol{\Phi})] .
\end{aligned}
$$

Если $\boldsymbol{\Phi}$ - постоянный вектор, то система (52) интегрируется в явном виде. В общем случае интегрирование в квадратурах невозможно. Отметим, что уравнение (23) имеет бесконечную последовательность скалярных $y$-интегралов с начальным элементом

$$
I=\mathbf{u}_{x x}^{2}-\frac{a\left(\mathbf{u}_{x}, \mathbf{u}_{x x}\right)^{2}}{1+a \mathbf{u}_{x}^{2}}, \quad D_{y} I=0 .
$$

7. Уравнение (24) является анизотропным аналогом уравнения (23). Оно имеет векторный $y$-интеграл вида (45), поэтому справедливы уравнения $\mathbf{u}_{x}=w \boldsymbol{\Phi}(x)$, $w_{y}=\sqrt{a+\left\langle\mathbf{u}_{y}, \mathbf{u}_{y}\right\rangle}, \boldsymbol{\Phi}^{2}=1$. Подстановка $\mathbf{u}_{y}=r \mathbf{v},\langle\mathbf{v}, \mathbf{v}\rangle=1$ приводит к системе обыкновенных уравнений

$$
r_{x}=\langle\mathbf{v}, \boldsymbol{\Phi}\rangle \sqrt{a+r^{2}}, \quad \mathbf{v}_{x}=r^{-1} \sqrt{a+r^{2}}[\mathbf{\Phi}-\mathbf{v}\langle\mathbf{v}, \boldsymbol{\Phi}\rangle],
$$

аналогичной (52). Уравнение (24), подобно уравнению (23), имеет бесконечную последовательность скалярных $x$-интегралов с начальным элементом

$$
\tilde{I}=\left\langle\mathbf{u}_{y y}, \mathbf{u}_{y y}\right\rangle-\frac{\left\langle\mathbf{u}_{y}, \mathbf{u}_{y y}\right\rangle^{2}}{a+\left\langle\mathbf{u}_{y}, \mathbf{u}_{y}\right\rangle}, \quad D_{x} \tilde{I}=0 .
$$

8. Уравнение (25) равносильно следующему уравнению:

$$
D_{y} \frac{\mathbf{u}_{x}}{\sqrt{a+\mathbf{u}^{2}}}=0
$$

и поэтому оно сводится к обыкновенному уравнению

$$
\mathbf{u}_{x}=\sqrt{a+\mathbf{u}^{2}} \boldsymbol{\Phi}(x)
$$

с произвольной вектор-функцией $\boldsymbol{\Phi}$.

9. Уравнение (26) имеет векторный $x$-интеграл

$$
D_{x}\left(\frac{\mathbf{u}_{y}}{\mathbf{u}^{2}+a}-\frac{(1+2 k q)\left(\mathbf{u}, \mathbf{u}_{y}\right)+b \eta}{r\left(\mathbf{u}^{2}+a\right)} \mathbf{u}\right)=0,
$$

поэтому оно сводится к следующему обыкновенному уравнению:

$$
\mathbf{u}_{y}-\frac{(1+2 k q)\left(\mathbf{u}, \mathbf{u}_{y}\right)+b \sqrt{\left(\mathbf{u}, \mathbf{u}_{y}\right)^{2}-r\left(\mathbf{u}_{y}^{2}+c q^{2}\right)}}{\mathbf{u}^{2}+k\left(\mathbf{u}^{2}+a\right)^{2}} \mathbf{u}=\left(\mathbf{u}^{2}+a\right) \mathbf{F}(y),
$$

где $r=\mathbf{u}^{2}+k\left(\mathbf{u}^{2}+a\right)^{2}, q=\mathbf{u}^{2}+a$. 


\section{4. ПРЕОБРАЗОВАНИЯ БЕКЛУНДА}

Преобразование Беклунда для эволюционного уравнения $u_{y}=K\left(u, u_{x}, \ldots, u_{x_{n}}\right)-$ это обыкновенное дифференциальное уравнение $\Phi\left(u, u_{x}, \ldots, u_{x_{k}}, v, v_{x}, \ldots, v_{x_{m}}\right)=0$, которое ставит в соответствие каждому решению $u$ исходного уравнения семейство решений $v$ того же уравнения. В этом случае преобразование Беклунда часто называют автопреобразованием Беклунда. Существуют также преобразования Беклунда, связывающие решения двух различных уравнений. Во многих случаях преобразования Беклунда - это уравнения первого порядка (см., например, [10]), но известны примеры преобразований Беклунда более высокого порядка (см., например, [11]); также известны преобразования Беклунда, зависящие от нелокальных переменных [12]. Преобразование Беклунда должно содержать произвольный параметр, который не устраняется каким-либо точечным преобразованием. Однако нам не известно определение понятия "существенной" зависимости от параметра.

В случае гиперболических уравнений $u_{x y}=f\left(u, u_{x}, u_{y}\right), v_{x y}=g\left(v, v_{x}, v_{y}\right)$ преобразование Беклунда первого порядка состоит из двух уравнений

$$
u_{x}=g\left(u, v, v_{x}\right), \quad u_{y}=h\left(u, v, v_{y}\right)
$$

(см., например, преобразование Беклунда для уравнения синус-Гордон в монографии [13]). Первое и второе уравнения (53) назовем $x$-компонентой и $y$-компонентой преобразования Беклунда соответственно. Попытка включить зависимость от $v_{y}$ в $x$-компоненту или зависимость от $v_{x}$ в $y$-компоненту приводит к противоречию. Таким образом, каждое из уравнений (53) - обыкновенное дифференциальное уравнение. Дифференцируя первое уравнение (53) по $y$, а второе по $x$ с учетом уравнений для $u$ и $v$, получаем два уравнения для определения функций $g$ и $h$. Из этих уравнений следует исключить производные $\partial_{x}^{k} u$ и $\partial_{y}^{k} u$ с помощью уравнений (53). Это дает два уравнения, которые должны выполняться тождественно по всем оставшимся переменным $u, v, v_{x}, v_{y}, v_{x x}, v_{y y}, \ldots$ В этом состоит главное отличие гиперболических уравнений от эволюционных, для которых можно ограничиться вычислением одной лишь $x$-компоненты преобразования Беклунда.

Известно, что для уравнения синус-Гордон $x$-компонента и $y$-компонента преобразования Беклунда являются преобразованиями Беклунда для уравнений мКд $\Phi$ $u_{t}=u_{x x x}+u_{x}^{3} / 2, u_{t}=u_{y y y}+u_{y}^{3} / 2$, являющихся $x$-симметриями и $y$-симметриями для уравнения синус-Гордон. Можно было бы предположить, что и для других гиперболических уравнений преобразования Беклунда составлены из двух преобразований для его симметрий, но это не всегда верно, как показано ниже.

С учетом изложенного введем преобразование Беклунда первого порядка для векторного уравнения (1) как два векторных обыкновенных уравнения:

$$
\mathbf{u}_{x}=g_{1} \mathbf{u}+g_{2} \mathbf{v}+g_{3} \mathbf{v}_{x}, \quad \mathbf{u}_{y}=h_{1} \mathbf{u}+h_{2} \mathbf{v}+h_{3} \mathbf{v}_{y},
$$

где $g_{1}, g_{2}, g_{3}$ - гладкие функции, зависящие от $\mathbf{u}^{2}, \mathbf{v}^{2},(\mathbf{u}, \mathbf{v}),\left(\mathbf{v}, \mathbf{v}_{x}\right), \mathbf{v}_{x}^{2}$ и $\left(\mathbf{u}, \mathbf{v}_{x}\right)$, a $h_{1}, h_{2}, h_{3}$ - гладкие функции, зависящие от $\mathbf{u}^{2}, \mathbf{v}^{2},(\mathbf{u}, \mathbf{v}),\left(\mathbf{v}, \mathbf{v}_{y}\right), \mathbf{v}_{y}^{2}$ и $\left(\mathbf{u}, \mathbf{v}_{y}\right)$. В анизотропном случае следует добавить еще шесть анизотропных переменных. При дифференцировании первого из уравнений (54) по $y$ и второго из уравнений (54) по $x$ 
в уравнениях появляются новые переменные

$$
\left(\mathbf{u}, \mathbf{u}_{y}\right),\left(\mathbf{v}, \mathbf{u}_{y}\right),\left(\mathbf{u}_{y}, \mathbf{v}_{x}\right),\left(\mathbf{u}, \mathbf{u}_{x}\right),\left(\mathbf{v}, \mathbf{u}_{x}\right),\left(\mathbf{u}_{x}, \mathbf{v}_{y}\right) .
$$

Все эти переменные, а также $\mathbf{u}_{x}^{2}$ и $\mathbf{u}_{y}^{2}$ вычисляются при помощи (54) и исключаются из уравнений. Затем уравнения расщепляются по переменным, не входящим в аргументы функций $g_{1}, g_{2}, g_{3}$ и $h_{1}, h_{2}, h_{3}$, и интегрируются. Ниже приведены автопреобразования Беклунда для гиперболических уравнений, которые не интегрируются в квадратурах. Параметр Беклунда везде обозначается как $\mu$.

Уравнение (16) имеет следующее автопреобразование Беклунда:

$$
\begin{aligned}
& \mathbf{u}_{x}-\mathbf{v}_{x}=2 \mu^{-1}\left[a\left(\mathbf{u}+\mathbf{v}, \mathbf{v}_{x}\right)+\xi \sqrt{1+a \mathbf{v}_{x}^{2}}\right](\mathbf{u}+\mathbf{v}), \\
& \mathbf{u}_{y}+\mathbf{v}_{y}=\frac{1}{2}(\mathbf{u}-\mathbf{v}) \xi, \quad \xi=\sqrt{\mu+a(\mathbf{u}+\mathbf{v})^{2}},
\end{aligned}
$$

где $x$-компонента преобразования (55) является преобразованием Беклунда для уравнения (10) (оно отличается от преобразования из работы [8] подстановкой $\lambda=$ $\left.a^{-1} \mu\right), y$-компонента есть преобразование Беклунда для векторного уравнения мКдФ (cм. [14])

$$
\mathbf{u}_{t}=\mathbf{u}_{y y y}-\frac{3}{2} a \mathbf{u}^{2} \mathbf{u}_{y},
$$

которое является $y$-симметрией уравнения (16).

Автопреобразование Беклунда для уравнения (17) запишем в следующем виде:

$$
\begin{gathered}
\mathbf{u}_{x}=G\left[\left(a \mu^{2} \frac{\left(\mathbf{v}_{x}, \mathbf{u}+\mathbf{v}\right)}{1+\gamma}+\mu\left(p_{v}-1\right)\right)(\mathbf{u}+\mathbf{v})+\mathbf{v}_{x}\right] \\
\mathbf{u}_{y}+\mathbf{v}_{y}=\frac{1}{\mu}(\gamma \mathbf{u}-\mathbf{v})+(1-\gamma) \frac{(\mathbf{u}+\mathbf{v}, \mathbf{v})}{\mu(\mathbf{u}+\mathbf{v})^{2}}(\mathbf{u}+\mathbf{v})
\end{gathered}
$$

где

$$
G=a \mu \frac{\left(\mathbf{v}_{x}, \mathbf{u}+\mathbf{v}\right)}{p_{v}-1}+\gamma, \quad p_{v}=\sqrt{1+a \mathbf{v}_{x}^{2}}, \quad \gamma=\sqrt{1+a \mu^{2}(\mathbf{u}+\mathbf{v})^{2}}
$$

Здесь $x$-компонента преобразования - это преобразование Беклунда для уравнения (12), а $y$-компонента - преобразование Беклунда для второго векторного уравнения мКдФ (см. [14])

$$
\mathbf{u}_{t}=\mathbf{u}_{y y y}-3 a\left(\mathbf{u}^{2} \mathbf{u}_{y}+\left(\mathbf{u}, \mathbf{u}_{y}\right) \mathbf{u}\right)
$$

которое является $y$-симметрией уравнения (17).

Автопреобразование Беклунда для уравнения (23) имеет следующий вид:

$$
\begin{aligned}
\mathbf{u}_{x}-\mathbf{v}_{x} & =2 \mu^{-1}\left[a\left(\mathbf{u}+\mathbf{v}, \mathbf{v}_{x}\right)+\xi \sqrt{1+a \mathbf{v}_{x}^{2}}\right](\mathbf{u}+\mathbf{v}), \\
\mathbf{u}_{y} & =-\left(1+\left|\mathbf{v}_{y}\right|^{-1} \xi\right) \mathbf{v}_{y}, \quad \xi=\sqrt{\mu+a(\mathbf{u}+\mathbf{v})^{2}} .
\end{aligned}
$$

Относительно $x$-компоненты см. комментарий к преобразованию (55), а $y$-компонента формально является преобразованием Беклунда для $y$-симметрии, которая 
имеет вид (11) при $a=0$. Но это преобразование не является настоящим преобразованием Беклунда для эволюционного уравнения, так как подстановка $\mathbf{u} \rightarrow \mathbf{u} \sqrt{\mu}$, $\mathbf{v} \rightarrow \mathbf{v} \sqrt{\mu}$ приводит к исчезновению параметра $\mu$ и не меняет вида эволюционного уравнения. Вид гиперболического уравнения меняется при указанной подстановке, поэтому $y$-компонента в (59) подходит на роль преобразования Беклунда для гиперболического уравнения.

Уравнение (24) имеет следующее автопреобразование Беклунда:

$$
\mathbf{u}_{x}=F_{1}\left(\mathbf{v}_{x}-2 f^{-1}\left(\mathbf{u}+\mathbf{v}, \mathbf{v}_{x}\right)(\mathbf{u}+\mathbf{v})\right), \quad \mathbf{u}_{y}=F_{2}(\mathbf{u}+\mathbf{v})-\mathbf{v}_{y},
$$

где

$$
\begin{aligned}
& F_{1}=\left|\mathbf{v}_{x}\right|^{-1} \sqrt{\mu f+g}-1, \quad f=(\mathbf{u}+\mathbf{v})^{2}, \quad g=\langle\mathbf{u}+\mathbf{v}, \mathbf{u}+\mathbf{v}\rangle, \\
& F_{2}=2(\mu f)^{-1}\left(\sqrt{\mu f+g} \sqrt{\left\langle\mathbf{v}_{y}, \mathbf{v}_{y}\right\rangle+a}-\left\langle\mathbf{u}+\mathbf{v}, \mathbf{v}_{y}\right\rangle\right) .
\end{aligned}
$$

Здесь $x$-компонента преобразования есть преобразование Беклунда для $x$-симметрии уравнения (14), а $y$-компонента не является преобразованием Беклунда для $S$-интегрируемой $y$-симметрии, которая получается из (10) заменой $a \rightarrow a^{-1},(\cdot, \cdot) \rightarrow$ $\langle\cdot, \cdot\rangle$. Благодаря наличию нетривиальных интегралов уравнение допускает бесконечное число $y$-симметрий, поэтому возможно, что $y$-компонента преобразования Беклунда совместна с какой-то из неканонических симметрий.

Автопреобразование Беклунда для уравнения (25) записывается в следующем виде:

$$
\begin{aligned}
& \mathbf{u}_{x}=\frac{a+(\mathbf{u}, \mathbf{v})-\eta}{a+\mathbf{v}^{2}} \mathbf{v}_{x}, \quad \eta=a+(\mathbf{u}, \mathbf{v})+\sqrt{\left(a+\mathbf{u}^{2}\right)\left(a+\mathbf{v}^{2}\right)} \\
& \mathbf{u}_{y}=\left[\frac{\left(\mathbf{u}, \mathbf{v}_{y}\right)}{\eta}-\frac{a+(\mathbf{u}, \mathbf{v})-\eta}{\eta\left(a+\mathbf{v}^{2}\right)}\left(\mathbf{v}, \mathbf{v}_{y}\right)-\mu \frac{a}{\sqrt{\eta}}\right](\mathbf{u}-\mathbf{v})+\mu \sqrt{\eta} \mathbf{u}+\mathbf{v}_{y} .
\end{aligned}
$$

Здесь $x$-компонента не является преобразованием Беклунда для $x$-симметрии уравнения, а $y$-компонента есть преобразование Беклунда для $y$-симметрии уравнения, которую можно получить из (10) при помощи потенциирования $\mathbf{u}_{y} \rightarrow \mathbf{u}$ и замены $a \rightarrow a^{-1}$.

Уравнение (26) имеет следующее автопреобразование Беклунда:

$$
\begin{aligned}
& \mathbf{u}_{x}=\frac{\mu}{\mathbf{v}_{x}^{2}}\left[(\mathbf{u}+\mathbf{v})^{2} \mathbf{v}_{x}-2\left(\mathbf{u}+\mathbf{v}, \mathbf{v}_{x}\right)(\mathbf{u}+\mathbf{v})\right] \\
& \mathbf{u}_{y}=\left[2 q_{0}^{-1}\left(\mathbf{u}+\mathbf{v}, \mathbf{v}_{y}\right)-\left(\mathbf{v}^{2}+2(\mathbf{u}, \mathbf{v})-a\right) h\right] \mathbf{u}+\left(\mathbf{u}^{2}+a\right) h \mathbf{v}-\frac{\mathbf{u}^{2}+a}{\mathbf{v}^{2}+a} \mathbf{v}_{y}
\end{aligned}
$$

где

$$
\begin{aligned}
& h=\frac{\left(1+2 k q_{0}\right)\left(\mathbf{v}, \mathbf{v}_{y}\right)+\zeta \sqrt{1+4 a k}}{q_{0}\left(k q_{0}^{2}+\mathbf{v}^{2}\right)}, \quad q_{0}=\mathbf{v}^{2}+a, \\
& \zeta=\sqrt{\left(\mathbf{v}, \mathbf{v}_{y}\right)-\left(\mathbf{v}_{y}^{2}+c q_{0}^{2}\right)\left(k q_{0}^{2}+\mathbf{v}^{2}\right)}
\end{aligned}
$$

Здесь $x$-компонента есть преобразование Беклунда для $x$-симметрии уравнения (15), а $y$-компонента не является преобразованием Беклунда ни для одной из $y$-симметрий уравнения. 


\section{5. ЗАКЛЮЧЕНИЕ}

В настоящей работе была исследована лишь малая часть известных интегрируемых эволюционных уравнений. Продолжение работы в этом направлении, без сомнения, позволит найти новые гиперболические векторные уравнения, обладающие высшими симметриями. Другое продолжение работы возможно на пути поиска интегрируемых гиперболических уравнений, допускающих симметрии более высокого порядка.

Все симметрии уравнений (16)-(26) являются $S$-интегрируемыми и имеют преобразования Беклунда. Но сами уравнения (16)-(26) принадлежат к трем типам: (А) уравнения (16), (17), преобразования Беклунда которых составлены из двух преобразований Беклунда для $x$ - и $y$-симметрий; (Б) - линеаризуемые уравнения (18)-(22); (В) - уравнения (23)-(26), приводящиеся к обыкновенным дифференциальным уравнениям.

Уравнения типа (А) наиболее близки по своим свойствам к уравнению синус-Гордон, и их можно, видимо, называть $S$-интегрируемыми. Множество уравнений типа (Б) можно разбить на два непересекающихся подмножества: $\left(\mathrm{Б}_{1}\right)$ - множество уравнений, приводящихся к уравнению Даламбера; $\left(\mathrm{Б}_{2}\right)$ - множество уравнений, приводящихся к другим линейным уравнениям. Уравнения из подмножества $\left(\mathrm{Б}_{1}\right)$ имеют нетривиальные интегралы по обеим характеристикам. Уравнения из подмножества $\left(\mathrm{Б}_{2}\right)$ имеют нетривиальные интегралы по одной характеристике, как, например, уравнение (22). Существуют линеаризуемые уравнения, не имеющие нетривиальных интегралов. К этому типу принадлежат, например, уравнения, приводимые к линейному уравнению Клейна-Гордона.

Для уравнений типа (В) характерны существование нетривиальных интегралов и частичная интегрируемость. Эти уравнения имеют преобразования Беклунда, но этим преобразованиям присущи некоторые дефекты (например, отсутствие параметра в одной из компонент), не позволяющие считать их настоящими преобразованиями Беклунда.

Рассмотрим кратко один из результатов статьи [6]. Уравнение

$$
\mathbf{u}_{x y}=-\frac{\left(\mathbf{u}_{y}, \mathbf{u}_{x}\right)}{\mathbf{u}^{2}+a} \mathbf{u}
$$

где $a$ - постоянная, авторы называют векторным уравнением синус-Гордон. Но для этого нет, как нам кажется, серьезных оснований. Наши вычисления показали, что уравнение (60) не имеет $S$-интегрируемых симметрий порядков $2,3,4$ и 5 . Представленная же в работе [6] симметрия является частным случаем следующей лиувиллевской $x$-симметрии:

$$
\mathbf{u}_{t}=\mathbf{u}_{x} F\left(I, D_{x} I, D_{x}^{2} I, \ldots\right),
$$

где $I=a \mathbf{u}_{x}^{2}+\left(\mathbf{u}, \mathbf{u}_{x}\right)^{2}-y$-интеграл уравнения (60), а $F$ - произвольная функция. Ввиду симметричности уравнения (60) функция $J=a \mathbf{u}_{y}^{2}+\left(\mathbf{u}, \mathbf{u}_{y}\right)^{2}$ является $x$-интегралом уравнения. По этому интегралу записывается $y$-симметрия того же типа, что и $x$-симметрия. 
Уравнение синус-Гордон, как известно, имеет бесконечный набор $S$-интегрируемых симметрий всех нечетных порядков, не больших 3 , и не имеет ни одного локального интеграла. Таким образом, свойства уравнений (60) и синус-Гордон совершенно различны.

Уравнение (60) скорее всего не является и $C$-интегрируемым (т.е. интегрируемым по Дарбу). Дело в том что оно не имеет векторных интегралов до четвертого порядка включительно, а все скалярные интегралы выражаются через $I, J$ и их производные.

Благодарности. Автор выражает благодарность участникам семинара по нелинейным уравнениям в ИТФ им. Л. Д. Ландау РАН за плодотворное обсуждение работы, а в особенности В.В. Соколову за постановку задачи и В.Э. Адлеру, указавшему на возможность существования векторных интегралов. Работа выполнена при финансовой поддержке Министерства образования и науки РФ (проект № 01.2.00705084).

\section{Список литературы}

[1] Н. Х. Ибрагимов, Группы преобразований в математической физике, Наука, М., 1983.

[2] П. Олвер, Приложения групп Ли к дифференииальным уравнениям, Мир, М., 1989.

[3] A. S. Fokas, Stud. Appl. Math., 77:3 (1987), 253-299.

[4] А. В. Михайлов, А. Б. Шабат, Р. И. Ямилов, УМН, 42:4(256) (1987), 3-53.

[5] А. В. Михайлов, А. Б. Шабат, В.В.Соколов, “Симметрийный подход к классификации интегрируемых уравнений", Интегрируемость и кинетические уравнения для солитонов, ред. В. Г. Бахтарьяр, В. Е. Захаров, В. М. Черноусенко, Наукова думка, Киев, 1990, 213-279; A. V. Mikhajlov, A. B. Shabat, V. V. Sokolov, "The symmetry approach to the classification of integrable equations", What is Integrability, Springer Ser. Nonlinear Dynam., Springer, Berlin, 1991, 115-184.

[6] S. C. Anco, T. Wolf, J. Nonlinear Math. Phys., 12, Suppl. 1 (2005), 13-31.

[7] А. В. Жибер, В. В. Соколов, УМН, 56:1(337) (2001), 63-106.

[8] А. Г. Мешков, В. В. Соколов, ТМФ, 139:2 (2004), 192-208.

[9] A. G. Meshkov, V. V. Sokolov, Comm. Math. Phys., 232:1 (2002), 1-18.

[10] R. M. Miura (ed.), Bäcklund Transformations, the Inverse Scattering Method, Solitons, and their Applications. NSF Research Workshop on Contact Transformations, Workshop on Contact Trans. (Vanderbilt Univ. Nashville, Tenn., September 27-29, 1974), Lecture Notes in Math., 515, Springer, Berlin, 1976.

[11] А.Г. Мешков, Фундамент. и прикл. матем., 12:7 (2006), 141-161.

[12] М. Ю. Балахнев, ТМФ, 154:2 (2008), 261-267.

[13] Дж. Л. Лэм, Введение в теорию солитонов, Мир, М., 1983.

[14] М. Ю. Балахнев, Матем. заметки, 82:4 (2007), 501-503.

Поступила в редакцию 30.04.2008, после доработки 15.04.2009 